A non-invasive method to find Langer's lines of skin.

rapportnr. WFW 92.055

Marcel Meuwissen

mei 1992

Stage artikel

Begeleiders: ir. M.R. van Ratingen

dr.ir. C.W.J. Oomens

Technische Universiteit Eindhoven

Vakgroep Fundamentele Werktuigkunde

Eindhoven, mei 1992 


\title{
A NON-INVASIVE METHOD TO FIND LANGER'S LINES OF SKIN
}

\author{
M.H.H. Meuwissen, M.R. van Ratingen, C.W.J. Oomens, J.D. Janssen \\ Eindhoven University of Technology \\ Eindhoven, The Netherlands
}

\section{SUMMARY}

The human skin is a very complex material: It has a high stiffness ratio, is time dependent and has inhomogeneous properties. One of these inhomogeneities is the fiber direction that varies with anatomical site. This fiber pattern is described by Langer's lines. In the present paper a numerical/experimental way for the determination of the fiber layout is used. The method is based on numerical analysis, strain distribution measurement and system identification. By means of a numerical simulation it is shown that it is possible to determine material parameters for an inhomogeneous material. For that, the skin is modeled as a linear elastic solid with a varying fiber direction. The favourable influence of a high stiffness ratio is demonstrated. Further the effects of noise and a modeling error on the estimation results are shown.

\section{INTRODUCTION}

Traditional experimental ways for the determination of mechanical properties lead to some problems when complex materials like skin are approached:

(1) A key element in traditional experiments is a homogeneous strain and stress distribution in at least a part of the sample. In an inhomogeneous material it is impossible to obtain this situation, unless extremely small specimen are used.

(2) The manufacturing of test samples deteriorates the internal coherence of the structure.

(3) Many experiments are necessary to measure all parameters for complex material models.

Hendriks $(1990,1991)$ proposed a numerical/experimental approach called the identification method. Its principle is shown in figure 1.

The sample in an arbitrary experiment is multi-axially loaded and the non-homogeneous strain distribution is registered with a position measurement system based on a digital image technique. A theoretical, finite element model is derived, based on this experiment. With a set of initial parameter estimates this model supplies computed strain data. These data are compared with the measured data, using the difference to adjust the initial parameter estimates. These new parameters are used in the mathematical model for another iteration and parameter adjustment. This way, the parameters in the model are estimated recursively, based on measured data. 


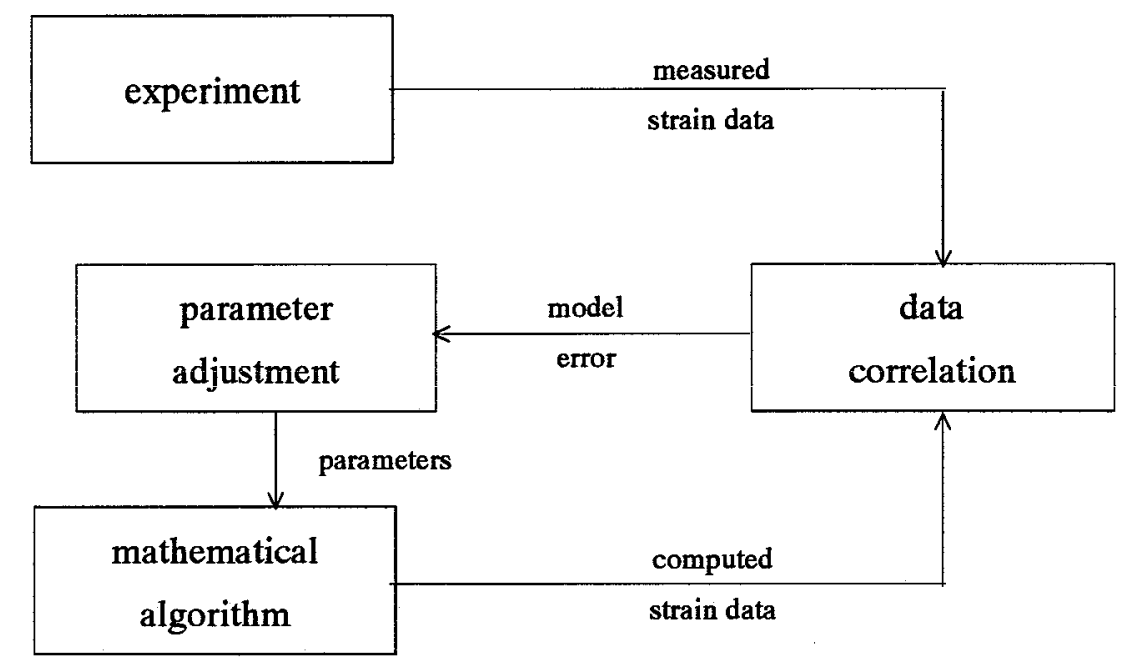

Figure 1: Diagram for the identification method.

For determining properties of skin, the measured data can be obtained by placing markers on a (living) subject in the region of interest for example the scapular region. Then this region is deformed. This can be done by external loading, for example by attaching some loading pad to the skin. By means of the markers, the strain field at the surface is registered. The displacements of the markers on the edge of the region can be used as boundary conditions in the finite element model. As a consequence forces cannot be part of the boundary conditions. This so-called local approach (Hendriks, 1991) does not allow the determination of absolute values of stiffness parameters but it is possible to determine stiffness ratios and an advantage is that the geometry of the model is relatively well defined.

The aim of the investigation presented in this paper is to examine the performance of the identification method in the parameter estimation of inhomogeneous materials. This is done by means of numerical simulations. Especially the influence of noise, different stiffness ratios and a modeling error are investigated.

The inhomogeneity in human skin that is the motive for these tests is the fiber orientation that varies from point to point. Langer's lines are lines that describe this fiber layout. The knowledge of these lines is particularly important for surgery, because incisions made in the Langer's lines leave fine linear scars while incisions at right angles to these lines result in widely stretched unsightly ones (Gray, 1973).

In the following section the theory of the identification algorithm is outlined. Then the numerical simulations will be described. Next the results are presented. Finally the results are discussed and some conclusions are drawn.

\section{THEORY}

In this section the identification method is described briefly. For more details we refer to Hendriks (1991). The algorithm is based on a sequential minimum variance approach. The nonlinear mathematical model is represented by:

$$
y=h(x)+v
$$


where $\mathbf{y}$ is a column containing the observed displacements, $\mathbf{x}$ is a set of material parameters, $\mathbf{h}$ describes the dependence of the observations $\mathbf{y}$ on $\mathbf{x}$, and $\mathbf{v}$ is a column of observation errors.

The iterative scheme, used to estimate $\mathbf{x}$ is given by:

$$
\begin{gathered}
\hat{\boldsymbol{x}}_{(i+1)}=\hat{\boldsymbol{x}}_{(i)}+\boldsymbol{K}_{(i+1)}\left(\boldsymbol{y}-\boldsymbol{h}\left(\hat{\boldsymbol{x}}_{(i)}\right)\right) \\
\boldsymbol{K}_{(i+1)}=\left(\boldsymbol{P}_{(i)}+\boldsymbol{Q}\right) \boldsymbol{H}_{(i+1)}^{T}\left(\boldsymbol{R}+\boldsymbol{H}_{(i+1)}\left(\boldsymbol{P}_{(i)}+\boldsymbol{Q}\right) \boldsymbol{H}_{(i+1)}^{T}\right)^{-1} \\
\boldsymbol{P}_{(i+1)}=\left(\boldsymbol{I}-\boldsymbol{K}_{(i+1)} \boldsymbol{H}_{(i+1)}\right)\left(\boldsymbol{P}_{(i)}+\boldsymbol{Q}\right)\left(\boldsymbol{I}-\boldsymbol{K}_{(i+1)} \boldsymbol{H}_{(i+1)}\right)^{T}+\boldsymbol{K}_{(i+1)} \boldsymbol{R} \boldsymbol{K}_{(i+1)}^{T}
\end{gathered}
$$

where $\mathrm{i}$ denotes the iterative step. The material parameters are updated in equation (2). Because the nonlinear function $\boldsymbol{h}\left(\hat{\boldsymbol{x}}_{\boldsymbol{i}}\right)$ symbolizes the finite element calculation with last parameter values, adjustment is based on the difference between newly calculated and the experimental data, the residual, multiplied by the updated gain matrix $\mathbf{K}_{(i+1)^{\circ}}$ This matrix is given by equation (3). The matrix $\mathbf{H}_{(i+1)}$ expresses the sensitivity of the model output for parameter variations and is defined as:

$$
\boldsymbol{H}_{(i+1)}=\left[\frac{\partial \boldsymbol{h}(\boldsymbol{x})}{\partial \boldsymbol{x}}\right]_{\boldsymbol{x}=\hat{\boldsymbol{x}}_{(i)}}
$$

To restrict the model's influence when parameter errors are large, the sum of squares is weighted with matrices $\mathbf{P}_{(i)}$ and $\mathbf{Q}$. In a sequential minimum variance estimator matrix $\mathbf{P}_{k}$ represents the covariance of the estimate $\hat{\boldsymbol{x}}_{(i)}$ and is given by:

$$
\boldsymbol{P}_{(i)}=E\left\{\left(\hat{\boldsymbol{x}}_{(i)}-E\left\{\hat{\boldsymbol{x}}_{(i)}\right)\right)\left(\hat{\boldsymbol{x}}_{(i)}-E\left\{\hat{\boldsymbol{x}}_{(i)}\right\}\right)^{T}\right\}
$$

In practice $\mathbf{Q}$ prevents that the parameter error covariance $\mathbf{P}_{(\mathbf{i})}$ becomes to small. The matrix $\mathbf{P}_{(\mathrm{i})}$ is updated in a special way by equation (4). It can be shown that in a linear case this will lead to an optimal or truly minimum variance estimator (best linear unbiased estimator).

The quality of the observations is expressed by the measurement error covariance matrix $\mathbf{R}$. By setting the matrix $\mathbf{R}$ the confidence in each separate displacement measurement can be indicated, so that more accurate measurements dominate the gain matrix. This tends to lead to faster convergence of material parameters.

The estimator (2) to (4) is implemented as an extra module PAREST in the finite element code DIANA used for finite element modeling (De Borst et al., 1985).

\section{NUMERICAL SIMULATIONS}

In practical situations the measured data are obtained from an experiment. In the simulations presented in this paper these data are generated artificially with a finite element model. An advantage of this choise is that the estimated results can be compared with exact values. Later the same model is reused to estimate the parameters.

First the properties of the material used in the simulations are given and the finite element 
model is described. We conclude this section with the estimation results.

\section{The model}

A curved region of the human body is modeled as a flat membrane (dimensions: $5 \times 4 \times 0.2$ ) which is shown in figure 2 . The membrane is assumed to have orthotropic properties. This means that the material has three mutually perpendicular planes of symmetry. Here we vary the orientation of these planes form point to point. One plane of symmetry coincides with the plane of the sample. The normal of one of the other planes is indicated in the figure with a short line which may be interpreted as the orientation of the fibers in the skin. The pattern shown in figure 2 closely resembles the fiber orientation (Langer's lines) in the skin on a cheek or the scapular region (Gray, 1973).

Further linear elasticity is assumed. This of course is a simplification, because the skin behaves nonlinear and is time dependent.

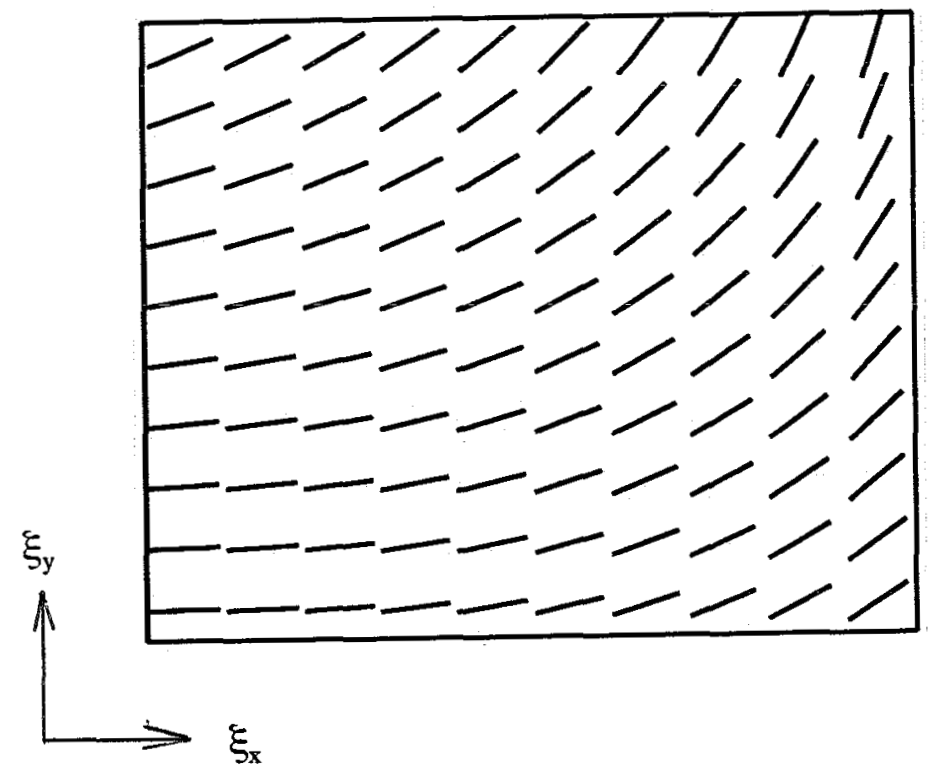

Figure 2: Sample shape and orientation of local planes of symmetry for $\alpha=0.02 \xi_{\mathrm{x}}+0.02 \xi_{\mathrm{y}}+0.02 \xi_{\mathrm{x}}^{2}+0.02 \xi_{\mathrm{y}}^{2}+0.02 \xi_{\mathrm{x}} \xi_{\mathrm{y}}$

In addition to showing the fiber orientation the Langer's lines are also lines of increased tension (Brown, 1971). The membrane in the simulations has no lines of increased tension. It is assumed that the fiber layout can be described with the following polynominal:

$$
\alpha=b_{1} \xi_{x}+b_{2} \xi_{y}+b_{3} \xi_{x}^{2}+b_{4} \xi_{y}^{2}+b_{5} \xi_{x} \xi_{y}
$$

where $\alpha$ denotes the positive rotation of the material 1-direction from the model $\xi_{\mathrm{x}}$-axis in radians. With equation (7) we introduced 5 material (fiber) parameters. The other parameters in the model are: the stiffness in the material 1- and 2-directions $E_{1}$ and $E_{2}$ respectively, the shear modulus $G_{12}$ and the Poison ratio $v_{21}$.

Figure 3 shows the finite element model used for the data generation and later for the parameters estimation. It consists of 100 bilinear plane stress elements. The nodal displacement in $\xi_{x}$-direction on the left and right edges and the $\xi_{y}$-displacements of the nodes 
on the top boundary are prescribed. The displacements in $\xi_{y}$-direction on the bottom edge are kept zero. The sample is loaded biaxially because it is expected that this situation will lead to the best performance of the estimation algorithm (Van Ratingen, 1992).

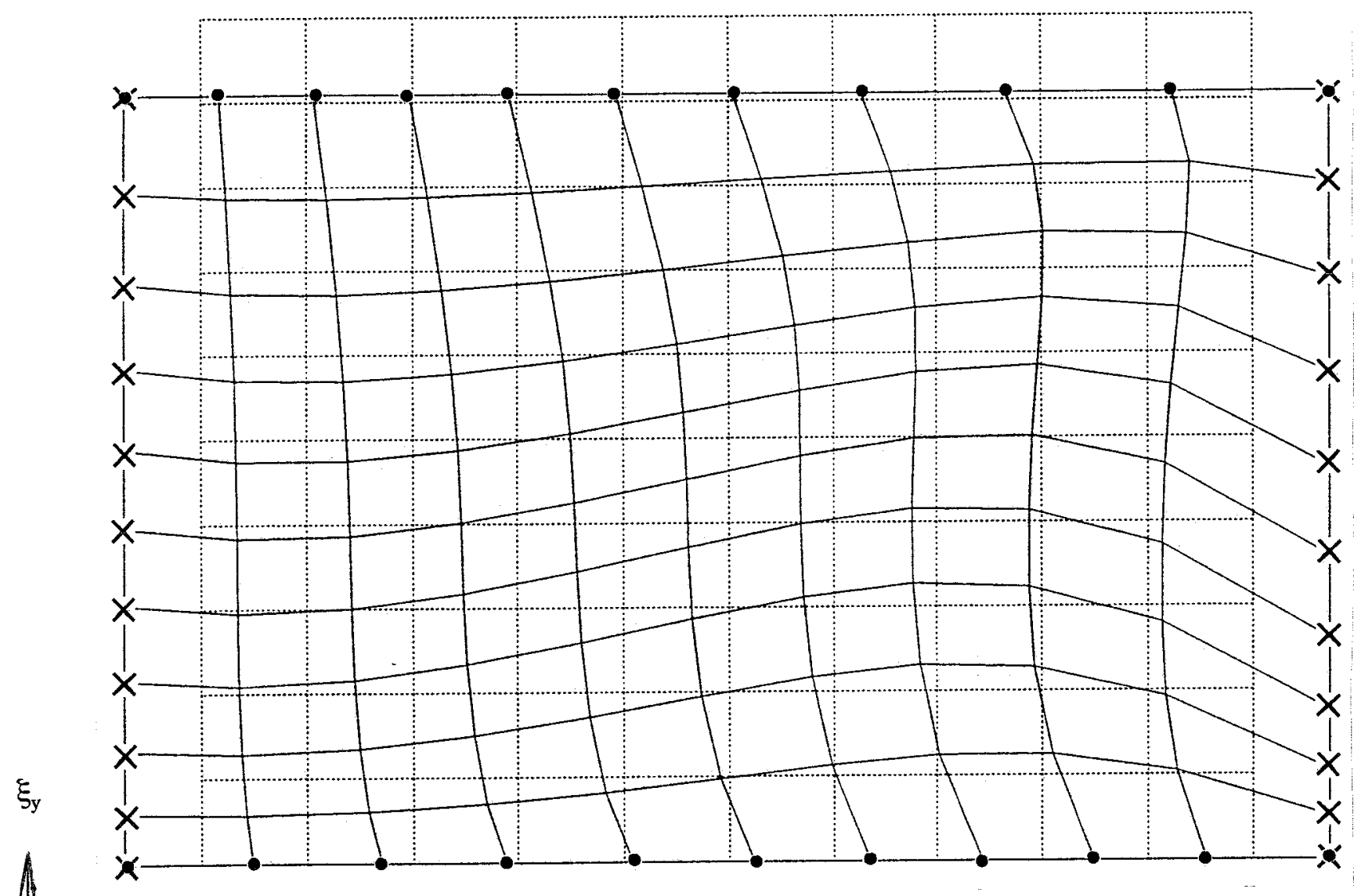

Figure 3: Finite element model: reference (dotted lines), loaded (solid lines).

$x:$ Prescribed $\xi_{\mathrm{x}}$ displacement

- : Prescribed $\xi_{\mathrm{y}}$ displacement

Notwithstanding the symmetrical load the deformation is clearly nonsymmetrical which is of course caused by the varying fiber direction.

The finite element model contains 121 nodes. The measured data consist of the $\xi_{x}-$ and $\xi_{y}-$ displacement components of the inner 81 nodal points.

\section{Results}

\section{Estimation of fiber parameters}

In this simulation only the parameters describing the fiber orientation in the sample will be estimated. The exact fiber layout, used for the generation of data, is given by:

$$
\alpha=0.02 \xi_{x}+0.02 \xi_{y}+0.02 \xi_{x}^{2}+0.02 \xi_{y}^{2}+0.02 \xi_{x} \xi_{y}
$$


This distribution is shown in figure 2. From initial guesses the parameters $b_{1}$ to $b_{5}$ must be determined. The parameter column $\mathbf{x}$ is defined by:

$$
\boldsymbol{x}^{T}=\left[b_{1}, b_{2}, b_{3}, b_{4}, b_{5}\right]
$$

The numerical values of the other parameters are chosen rather arbitrarily, although for an orthotropic material the parameters have to satisfy the following expression:

$$
v_{21} v_{12}=v_{21}^{2} \frac{E_{1}}{E_{2}}<1
$$

The test is performed for two different sets of material properties:

$$
\begin{aligned}
& \text { (1) } \mathrm{E}_{1}=1.0, \mathrm{E}_{2}=0.50, v_{21}=0.3, \mathrm{G}_{12}=0.50, \text { stiffness ratio } \mathrm{E}_{1} / \mathrm{E}_{2}=2 \\
& \text { (2) } \mathrm{E}_{1}=1.0, \mathrm{E}_{2}=0.10, v_{21}=0.3, \mathrm{G}_{12}=0.10, \text { stiffness ratio } \mathrm{E}_{1} / \mathrm{E}_{2}=10
\end{aligned}
$$

In both situations an estimation is performed with exact "measured" data and with realizations of a zero mean uniform random distribution added to these data. In some simulations this noise is also added to the boundary conditions of the model. The standard deviation of the

\begin{tabular}{|c|c|c|c|c|c|c|c|c|}
\hline \multirow[t]{4}{*}{ Parameter } & \multirow{4}{*}{$\begin{array}{l}\text { Exact } \\
\text { value }\end{array}$} & \multirow{4}{*}{$\begin{array}{l}\text { Initial } \\
\text { guess }\end{array}$} & \multicolumn{6}{|c|}{ Estimations } \\
\hline & & & \multicolumn{3}{|c|}{$\mathrm{E}_{1} / \mathrm{E}_{2}=\mathbf{2 . 0}$} & \multicolumn{3}{|c|}{$\mathrm{E}_{1} / \mathrm{E}_{2}=10.0$} \\
\hline & & & \multicolumn{2}{|c|}{ No modeling error } & \multirow{2}{*}{$\begin{array}{c}\begin{array}{c}\text { Modeling } \\
\text { error }\end{array} \\
\sigma=10^{4}\end{array}$} & \multicolumn{2}{|c|}{ No modeling error } & \multirow{2}{*}{$\begin{array}{c}\begin{array}{c}\text { Modeling } \\
\text { error }\end{array} \\
\sigma=10^{-4}\end{array}$} \\
\hline & & & No noise & $\sigma=10^{-4}$ & & No noise & $\sigma=10^{-4}$ & \\
\hline$b_{1}$ & 0.02 & 0.01 & 0.02000 & 0.01951 & 0.01440 & 0.02000 & 0.01997 & 0.01893 \\
\hline$b_{2}$ & 0.02 & 0.04 & 0.02000 & 0.02001 & 0.02609 & 0.02000 & 0.02019 & 0.02200 \\
\hline$b_{3}$ & 0.02 & 0.00 & 0.02000 & 0.02014 & 0.02128 & 0.02000 & 0.02000 & 0.02016 \\
\hline$b_{4}$ & 0.02 & 0.00 & 0.02000 & 0.02008 & 0.01892 & 0.02000 & 0.01995 & 0.01901 \\
\hline$b_{s}$ & 0.02 & 0.00 & 0.02000 & 0.01987 & 0.01919 & 0.02000 & 0.01999 & 0.02017 \\
\hline \multicolumn{3}{|c|}{ Number of iterative steps: } & 9 & 10 & 10 & 9 & 9 & 9 \\
\hline
\end{tabular}
noise is $10^{-4}$, which leads to a noise signal ratio of slightly less than $1 \%$. The added noise to the boundary conditions can be regarded as a modeling error. This error is not accounted for in the estimation algorithm.

Table 1: Estimation results.

The initial estimate of the matrix $\mathbf{P}$ is considered to be diagonal:

$$
\boldsymbol{P}_{0}=\left\lceil 10^{-3}, 10^{-3}, 10^{-3}, 10^{-3}, 10^{-3}\right\rfloor
$$


The matrix $\mathbf{R}$ is also considered diagonal. The diagonal elements equal $\sigma^{2}=10^{-4}$ for the cases where the disturbed data are used. When no noise is added to the data $\mathbf{R}$ is chosen small but not zero, because this causes numerical problems. Table 1 gives the initial guesses for the parameters and the estimation results.

\section{Mixed estimations}

The previous simulations were not very realistic because in many experiments not only the fiber parameters but also stiffness parameters and Poison's ratio are unknown. Therefore, in this test, the identification method's ability to estimate a "mixed" (i.e. material and fiber) set of parameters is investigated. The exact fiber layout is given by:

$$
\alpha=0.1 \xi_{x}+0.1 \xi_{y}
$$

This distribution is shown in figure 4. The estimated parameters are: the fiber parameters $b_{1}$ and $b_{2}$, stiffness $E_{2}$ and Poison's ratio $v_{21}$ :

$$
\boldsymbol{x}^{T}=\left[b_{1}, b_{2}, E_{2}, v_{21}\right]
$$

The stiffness in the material 1-direction and the shear modulus are assumed to be known:

$$
\mathrm{E}_{1}=1.0, \mathrm{G}_{12}=0.25
$$

Again the cases with and without noise added to the measured data and the model's boundary conditions are analysed. The noise has a zero mean uniform random distribution with a standard deviation of $\sigma=10^{-4}$, which results in a noise signal ratio of nearly $1 \%$.

The estimate of the matrix $\mathbf{P}_{0}$ is given by:

$$
\boldsymbol{P}_{0}=\left\lceil 10^{-2}, 10^{-2}, 10^{-1}, 10^{-2}\right\rfloor
$$

Note that less confidence is expressed in the initial estimate of $E_{2}$ by choosing the corresponding diagonal element in $\mathbf{P}_{0}$ bigger. The choises of $\mathbf{R}$ remain unchanged. The exact values as well as the initial guesses of the parameters and the estimation results are given in table 2 .

\begin{tabular}{|c|c|c|c|c|c||}
\hline \multirow{2}{*}{ Parameter } & \multirow{2}{*}{ Exact value } & \multirow{2}{*}{ Initial guess } & \multicolumn{3}{|c|}{ Estimations } \\
\cline { 4 - 6 } & & & \multicolumn{2}{|c|}{ No modeling error } & Modeling error \\
\cline { 4 - 6 } & & & No noise & $\sigma=10^{-4}$ & $\sigma=10^{-4}$ \\
\hline$b_{1}$ & 0.10 & 0.01 & 0.1000 & 0.1001 & 0.1001 \\
$b_{2}$ & 0.10 & 0.04 & 0.1000 & 0.0999 & 0.0973 \\
$E_{2}$ & 0.25 & 0.50 & 0.2500 & 0.2500 & 0.2471 \\
$v_{12}$ & 0.30 & 0.20 & 0.3000 & 0.3043 & 0.2996 \\
\hline \multicolumn{2}{|c|}{ Number of iterative steps: } \\
\hline
\end{tabular}

Table 2: Estimation results. 


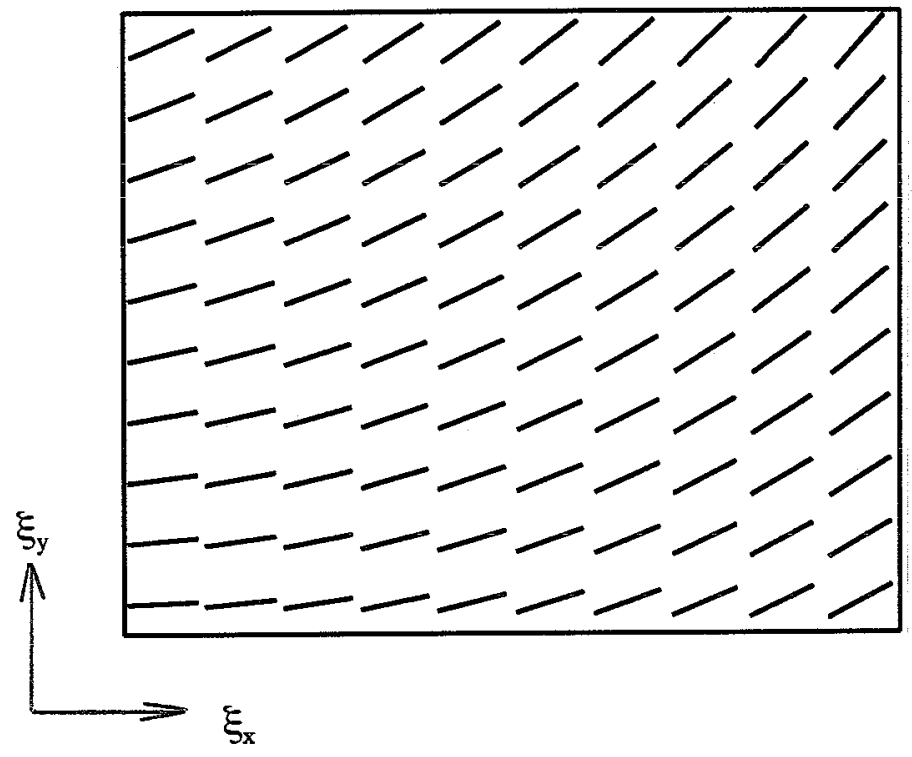

Figure 4: Sample shape and orientation of local planes of symmetry for $\alpha=0.1 \xi_{x}+0.1 \xi_{y}$

\section{DISCUSSION AND CONCLUSIONS}

The material used in the simulations is a gross simplification of human skin. We assumed linear elasticity while the skin is nonlinear and in addition time dependent. Further the curved skin surface is modeled as a flat membrane and the influence of the lines of increased tension is neglected. Nevertheless these restrictions are made because the aim of the simulations was the examination of the identification method for parameter estimation of inhomogeneous materials like skin.

The fiber layout of the skin in the regions of interest can be well described using the parameters $b_{1}$ and $b_{2}$ only. Yet, we used five parameters to test if the measured data contain enough information to give good estimates of this larger set of parameters.

In all simulations the parameter estimates converged to a stationary value. Comparing the exact parameter values with the estimated ones, it can be concluded that it is indeed possible to characterize a material with a varying fiber direction using the identification method.

Adding noise to the "measured" data causes parameter estimates to deviate from the exact value, as expected. Note that a material with a higher stiffness ratio $E_{1} / E_{2}$ is less influenced by noise. This can be explained as follows: A stiffness ratio of 1 leads to nearly isotropic (and thus, in our simulations, to nearly homogeneous) material behavior. A stiffness ratio that differs slightly from 1 results in a displacement field that is nearly the same as the displacement field for a homogeneous material. This little difference leads to an overparameterized model which is very sensible to noise and as a consequence finding good parameter estimates is difficult. A larger stiffness ratio leads to a displacement field that differs considerably from the homogeneous displacement field and this field is thus less influenced by noise. 
The introduction of the (small) modeling error has a significant impact on the estimation results. In some cases (see for example the situation $\mathrm{E}_{1} / \mathrm{E}_{2}=2.0$ ) the deviation from the exact value can rise up to $30 \%$. It can be observed that this estimation error is also influenced by the stiffness ratio: a higher stiffness ratio gives better results.

Further it can be noted that the estimation results of the mixed parameter set is better than the estimation results of the fiber parameters only. A possible explanation is that the fiber layout in the mixed situation is much simpler: It can be described with two parameters only, while in the other case the fiber pattern is described with five parameters.

To make the method applicable to human skin, some further extensions have to be made. The skin has to be modeled more realistic, so instead of linear elastic material behavior, nonlinear time dependent material behavior should be implemented in the model. Further, in the finite element model the flat membrane has to be replaced by a more realistic curved surface. Finally experiments on the skin require a 3-dimensional displacement measurement technique.

\section{REFERENCES}

De Borst, R., Kusters, G.M.A., Nanta, P., De Witte, F.C., 1985, "DIANA - A comprehensive, but flexible finite element system", in: Finite Element Systems: A Handbook,ed. C.A. Brebbia, Springer Verlag, Berlin, New York and Tokyo.

Brown, I.A., 1971, "Structural aspects of the biomechanical properties of human skin", Ph.D.-thesis, University of Strathclyde, Glasgow.

Hendriks, M.A.N., 1991, "Identification of the mechanical behaviour of solid materials", Ph.D.-thesis, Eindhoven University of Technology, The Netherlands.

Hendriks, M.A.N., Oomens, C.W.J., Janssen, J.D., 1991, "A Hybrid method to determine material parameters of composites", In:Mechanical Identification of Composites, ed. E. Vautrin, H. Sol, Elsevier Applied Science, pp.75-81.

Hendriks, M.A.N., Oomens, C.W.J., Jans, H.W.J., Janssen, J.D., Kok, J.J., 1990, "A numerical experimental approach for the mechanical characterization of composites", Proc. 9th Int. Conf. on Exp. Mech. Kopenhagen, pp. 552-561.

Gray, H., 1973, "Anatomy of the human body", 29th American Edition, ed. Lea and Febinger, Philadelphia, ISBN 0-8121-0377-7.

Langer, A.K., 1861, "Zur Anatomie und Physiologie der Haut. 1. Über die Spaltbarkeit der Cutis", S.B. der Akad, Vienna.

Ratingen, M.R. van, 1992, "Identification of orthotropic membranes with high stiffness ratio", technical report, Institute for Continuing Education, Eindhoven University of Technology, The Netherlands. 